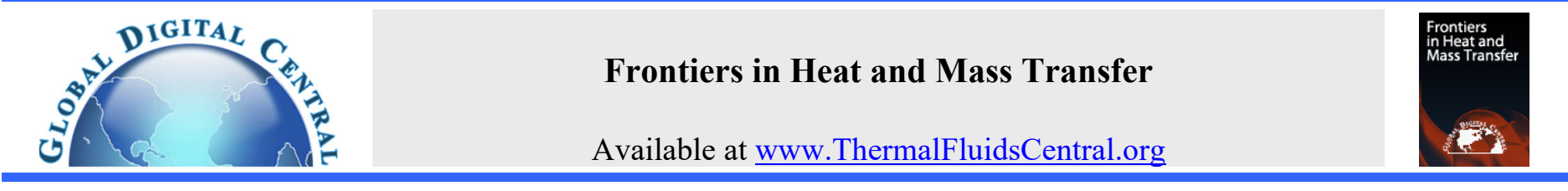

\title{
INFLUENCE OF MHD ON FREE CONVECTION OF NON-NEWTONIAN FLUIDS OVER A VERTICAL PERMEABLE PLATE IN POROUS MEDIA WITH INTERNAL HEAT GENERATION
}

\author{
Heng-Pin Hsu ${ }^{\mathrm{a}}$, Chuo-Jeng Huang ${ }^{\mathrm{b} *}$, Herchang $\mathrm{Ay}^{\mathrm{a}}$ \\ ${ }^{a}$ Department of Mold and Die Engineering, National Kaohsiung University of Science and Technology (R.O.C.) \\ ${ }^{b}$ Department of Aircraft Engineering, Air Force Institute of Technology, Taiwan (R.O.C.)
}

\begin{abstract}
The heat and mass transfer characteristics of the influence of uniform blowing/suction and MHD (magnetohydrodynamic) on the free convection of non-Newtonian fluids over a vertical plate in porous media with internal heat generation and Soret/Dufour effects are numerically analyzed. The surface of the vertical plate has a uniform wall temperature and uniform wall concentration (UWT/UWC). The numerical modeling of this problem attracts considerable attention, owing to its practical applications in biological sciences, electronic cooling, advanced nuclear systems, etc. The transformed governing equations are solved by Keller box method. Comparisons showed excellent agreement with the numerical data in previous works. Numerical data for the dimensionless temperature profile, the dimensionless concentration profile, the local Nusselt number and the local Sherwood number are presented for the main parameters: the magnetic field parameter $M$, the blowing/suction parameter $\xi$, the power-law index of the non-Newtonian fluid $n$ and the internal heat generation $A^{*}$. The physical aspects of the problem are discussed in details.

Keywords: uniform blowing/suction, MHD, non-Newtonian fluid, Soret/Dufour effects, internal heat generation, free convection, vertical plate, porous medi
\end{abstract}

\section{INTRODUCTION}

The boundary-layer flow of coupled heat and mass transfer (or doublediffusion) of non-Newtonian fluids in a porous media in which the main driving force is gravity has attracted a large class of applications in engineering practice, particularly in applied geophysics, geology, groundwater flow and oil reservoir engineering. These include nuclear reactor cooling system, extraction of geothermal energy, thermal insulation of buildings, filtration processes and disposal of underground nuclear wastes. Due to the increase in the production of heavy crude oils, and elsewhere where materials whose flow behavior in shear cannot be characterized by Newtonian relationships, it has become necessary to have an adequate understanding of the effects of nonNewtonian fluid flows and, as a result, a new stage in the evolution of fluid dynamic theory is in progress. Nield and Bejan (2006) recently presented a comprehensive account of the available information in the field.

In particular, a number of industrially important fluids including fossil fuels exhibit non-Newtonian fluid behavior. Non-Newtonian power law fluids are so widespread in industrial processes and in the environment that it would be no exaggeration to affirm that Newtonian shear flows are the exception rather than the rule. Shenoy (1994) presented many interesting applications of non-Newtonian power law fluids with yield stress on convective heat transport in fluid saturated porous media considering geothermal and oil reservoir engineering applications. In the aspect of pure heat transfer, Chen and Chen (1988) presented similarity solutions for natural convection of a nonNewtonian fluid over vertical surfaces in porous media. Yang and Wang (1996) investigated the natural convection heat transfer of nonNewtonian power-law fluids with yield stress over axisymmetric and two-dimensional bodies of arbitrary shape embedded in a fluid- saturated porous medium. Gorla and Kumari (1999) studied nonsimilar solutions for free convection in non-Newtonian fluids along a vertical plate in a porous medium. In the aspect of coupled heat and mass transfer, the double-diffusion from a vertical surface in a porous region saturated with a non-Newtonian fluid has been treated by Rastogi and Poulikakos (1995). Jumah and Mujumdar (2000) examined the natural convection heat and mass transfer from a vertical plate with variable wall temperature and concentration to power law fluids with yield stress in a porous medium. Cheng (2006) studied the natural convection heat and mass transfer of non-Newtonian power law fluids with yield stress in porous media from a vertical plate with variable wall heat and mass fluxes. Tai and Char (2010) examined the Soret and Dufour effects on free convection flow of non-Newtonian fluids along a vertical plate embedded in a porous medium with thermal radiation. Yih and Huang (2015) reported the effect of internal heat generation on free convection flow of non-Newtonian fluids over a vertical truncated cone in porous media: VWT/VWC.

The Soret effect referred to species differentiation developing in an initial homogeneous mixture submitted to a thermal gradient. The Dufour effect referred to heat flux produced by a concentration gradient. Postelnicu (2004) examined the heat and mass characteristics of natural convection about a vertical surface embedded in a saturated porous medium subjected to a magnetic field by considering the Dufour and Soret effects. Partha et al. (2006) studied the Soret and Dufour effects in a non-Darcy porous medium. Cheng (2007) examined the Soret and Dufour effects on free convection boundary layers of non-Newtonian power law fluids with yield stress in porous media over a vertical plate with variable wall heat and mass fluxes. Hsiao et al. (2014) investigated the influence of thermophoretic particle deposition on MHD free convection flow of non-Newtonian fluids from a vertical plate embedded in porous media considering Soret and Dufour effects.

*Corresponding author. Email: hcj631216@yahoo.com.tw 
The effect of internal heat generation is important in several applications that include reactors safety analyses, metal waste form development for spent nuclear fuel, fire and combustion studies, and the storage of radioactive materials. A new class of similarity solutions has obtained for isothermal vertical plate in a semi-infinite quiescent fluid with internal heat generation decaying exponentially by Crepeau and Clarksean (1997). Postelnicu and Pop (1999) studied the similarity solutions of free convection boundary layers over vertical and horizontal surfaces in porous media with internal heat generation. Grosan and Pop (2001) examined a free convection over vertical flat plate with a variable wall temperature and internal heat generation in a porous medium saturated with a non-Newtonian fluid. Grosan et al. (2009) reported magnetic field and internal heat generation effects on the free convection in a rectangular cavity filled with a porous medium.

However, in the above paper, these scholars are concentrated upon the impermeable case. Minkowycz and Cheng (1982) reported local non-similar solutions for free convective flow with uniform lateral mass flux in porous medium. Yih (1997) examined the effect of uniform lateral mass flux on free convection about a vertical cone embedded in a saturated porous medium. Yih (1998) investigate the effect of uniform lateral mass flux on the heat transfer characteristics in natural convection of non-Newtonian fluids in a saturated porous medium. Chamkha and Ben-Nakhi (2008) studied the MHD mixed convectionradiation interaction along a permeable surface immersed in a porous medium in the presence of Soret and Dufour's effects. Kumari and Nath (2009) studied natural convection from a vertical cone in a porous medium due to the combined effects of heat and mass diffusion with non-uniform wall temperature/concentration or heat/mass flux and suction/injection. Rashad et al. (2011) examined natural convection boundary layer of a non-Newtonian fluid about apermeable vertical cone embedded in a porous medium saturated with a nanofluid. Huang (2017) investigated the influence of uniform blowing/suction on the free convection of non-Newtonian fluids over a vertical cone in porous media with thermal radiation and Soret/Dufour effects: uniform wall temperature/uniform wall concentration. Huang (2018) examined the effects of internal heat generation and Soret/Dufour on natural convection of non-Newtonian fluids over a vertical permeable cone in a porous medium.

The aim of the present work, therefore, is to extend the work of Tai and Char (2010) to investigate the influence of uniform blowing/suction on the free convection of non-Newtonian fluids over a vertical plate embedded in porous media with internal heat generation and Soret/Dufour effect. The governing equations have been solved numerically using Keller box method (KBM). The results are obtained for various values of the parameters.

\section{ANALYSIS}

The considered problem is the influence of uniform blowing/suction on the natural convection of non-Newtonian fluids over a vertical plate in porous media with internal heat generation and Soret/Dufour effect and where the boundary condition is uniform wall temperature $T_{w}$ and uniform wall concentration $C_{w}$ (UWT/UWC), respectively. Consider a two-dimensional, steady, laminar flow of an incompressible electrically conducting fluid over a flat plate in the presence of a transverse magnetic field $B_{0}$, as shown in Fig. 1, while the induced magnetic field due to the motion of the electrically conducting fluid is negligible. The origin of the coordinate system is the leading edge of the vertical flat plate, where $x$ and $y$ are Cartesian coordinates for the distance along and normal to, respectively, the vertical flat plate surface.

All fluid properties are assumed to be constant except for the density variation in the buoyancy term. Introducing the boundary layer and Oberbeck-Boussinesq approximations, the governing equations and the boundary conditions based on the Darcy law (It is valid under the condition of low velocity and small pores of porous medium [31]) can

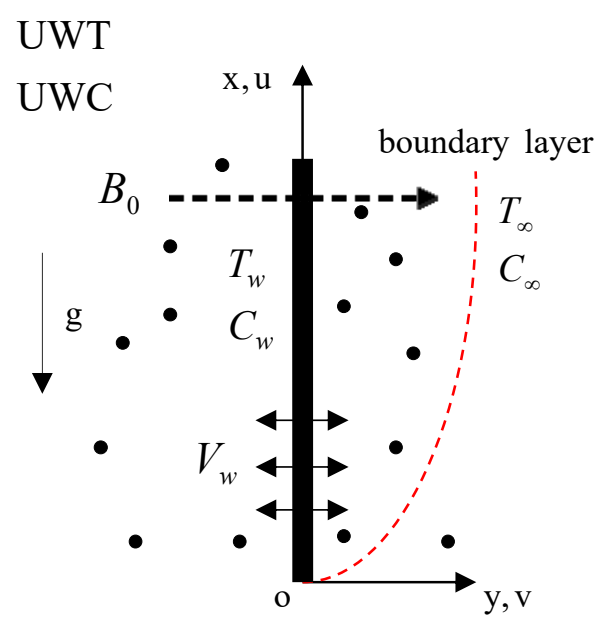

Fig. 1 The flow model and the physical coordinate system

be written as follows:

Continuity equation:

$\frac{\partial u}{\partial x}+\frac{\partial v}{\partial y}=0$

Momentum (Darcy) equation:

$u^{n}+\frac{K(n) \cdot \sigma \cdot B_{0}^{2}}{\mu} \cdot u=-\frac{K(n)}{\mu}\left(\frac{\partial p}{\partial x}+\rho g\right)$

$v^{n}=-\frac{K(n)}{\mu}\left(\frac{\partial p}{\partial y}\right)$

Energy equation:

$u \frac{\partial T}{\partial x}+v \frac{\partial T}{\partial y}=\alpha_{m} \frac{\partial^{2} T}{\partial y^{2}}+\bar{D} \frac{\partial^{2} C}{\partial y^{2}}+\frac{q^{\prime \prime \prime}}{\rho \cdot C_{p}}$

Concentration equation:

$u \frac{\partial C}{\partial x}+v \frac{\partial C}{\partial y}=D_{M} \frac{\partial^{2} C}{\partial y^{2}}+\bar{S} \frac{\partial^{2} T}{\partial y^{2}}$

Boussinesq approximation:

$\rho=\rho_{\infty}\left[1-\beta_{T}\left(T-T_{\infty}\right)-\beta_{C}\left(C-C_{\infty}\right)\right]$

Boundary conditions:

$y=0: v=V_{w}, T=T_{w}, C=C_{w}$

$y \rightarrow \infty: u=0, T=T_{\infty}, C=C_{\infty}$

Here, $u$ and $v$ are the Darcian velocities in the $x$ - and $y-$ directions, respectively; $n$ is the power-law index of the nonNewtonian fluid; $K(n)$ is the permeability of the porous medium; $\sigma$ is the electric conductivity of the fluid; $B_{0}$ is the externally imposed magnetic field in the $y$-direction; $g$ is the gravitational acceleration; $p, \rho$ and $\mu$ are the pressure, the density and the absolute viscosity, respectively; $T$ and $C$ are the volume-averaged temperature and concentration, respectively; $\alpha_{m}$ and $D_{M}$ are the equivalent thermal diffusivity and mass diffusivity, respectively; $C_{p}$ is the specific heat at constant pressure; $q^{\prime \prime \prime}$ is the internal heat generation rate per unit volume; $T_{m}$ is the mean fluid temperature; $\beta_{T}$ and $\beta_{C}$ are the thermal and concentration expansion coefficients of the fluid, respectively; $V_{w}$ is the uniform blowing/suction velocity.

The second term on the right-hand side of the energy Eq. (4) represents the Dufour effect, and the last term of concentration Eq. (5) denotes the Soret effect.

The power-law fluid index $n$ for various fluids is as follows: 
(i) $n<1$ for pseudo-plastic fluids (for example, the polymer solution) or shear-thinning fluids that have a lower apparent viscosity at higher shear rates.

(ii) $n=1$ for Newtonian fluids (for instance, air and water) where the shear stress is directly proportional to the shear rate.

(iii) $n>1$ for dilatant fluids (for example, the suspensions of sand) or shear-thickening fluids for which there is an increase in the apparent viscosity at higher shear rates.

For the power law model of Ostwald-de-Waele, Christopher and

Middleman (1965) and Dharmadhikari and Kale (1985) proposed the following relationships for the permeability:

$K(n)=\left\{\begin{array}{l}\frac{6}{25}\left(\frac{n \varepsilon}{3 n+1}\right)^{n}\left[\frac{\varepsilon d}{3(1-\varepsilon)}\right]^{n+1} \\ \frac{2}{\varepsilon}\left[\frac{d \varepsilon^{2}}{8(1-\varepsilon)}\right]^{n+1}\left(\frac{6 n+1}{10 n-3}\right)\left(\frac{16}{75}\right)^{\frac{3(10 n-3)}{(10 n+11)}}\end{array}\right.$

where $d$ is the particle diameter and $\varepsilon$ is the porosity.

The stream function $\psi$ is defined by

$u=\partial \psi / \partial y$ and $v=-\partial \psi / \partial x$

Therefore, the continuity equation is automatically satisfied.

Next, consider governing Eqs. (2) and (3). Cross-differentiation $\partial\left(u^{n}\right) / \partial y-\partial\left(v^{n}\right) / \partial x$, eliminates the pressure terms in Eqs. (2) and (3).

The boundary layer approximation $(\partial / \partial x<<\partial / \partial y, v<<u)$ is then used to obtain:

$\frac{\partial u^{n}}{\partial y}+\left(\frac{K(n) \cdot \sigma \cdot B_{0}^{2}}{\mu}\right) \cdot \frac{\partial u}{\partial y}=\frac{\rho \cdot g \cdot K(n)}{\mu} \cdot\left(\beta_{T} \frac{\partial T}{\partial y}+\beta_{C} \frac{\partial C}{\partial y}\right)$

Integrating Eq. (11) once and with the aid of boundary equation (8),

$u^{n}+\left(\frac{K(n) \cdot \sigma \cdot B_{0}^{2}}{\mu}\right) \cdot u=\frac{\rho \cdot g \cdot K(n)}{\mu}\left[\beta_{T}\left(T-T_{\infty}\right)+\beta_{C}\left(C-C_{\infty}\right)\right]$

The following dimensionless variables are invoked:

$$
\begin{aligned}
& \xi=\frac{2 V_{w} x}{\alpha_{m} \cdot R a_{x}^{1 / 2 n}} \\
& \eta=\frac{y}{x} R a_{x}^{1 / 2 n} \\
& f(\xi, \eta)=\frac{\psi}{\alpha_{m} \cdot R a_{x}^{1 / 2 n}} \\
& \theta(\xi, \eta)=\frac{T-T_{\infty}}{T_{w}-T_{\infty}} \\
& \phi(\eta, \xi)=\frac{C-C_{\infty}}{C_{w}-C_{\infty}} \\
& R a_{x}=\frac{\rho \cdot g \cdot \beta_{T} \cdot\left(T_{w}-T_{\infty}\right) \cdot K(n)}{\mu} \cdot\left(\frac{x}{\alpha_{m}}\right)^{n}
\end{aligned}
$$

where $R a_{x}$ is the local Rayleigh number.

Following Postelnicu and Pop (1999), the internal heat generation rate per unit volume $q^{\prime \prime \prime}$ is modeled according to the following equation:

$q^{\prime \prime \prime}=A^{*} \cdot \frac{k \cdot R a_{x}^{1 / n}}{x^{2}} \cdot\left[T_{w}-T_{\infty}\right] \cdot e^{-\eta}$

Here, $A^{*}$ is the internal heat generation coefficient. Note that, when $A^{*}=0$, this case corresponds to the absence of internal heat generation while, when $A^{*}>0$, this case corresponds to the presence of internal heat generation.

Substituting Eq. (13) into Eqs. (12), (4)-(5), (7)-(8) obtains

$\left(f^{\prime}\right)^{n}+M \cdot\left(f^{\prime}\right)=\theta+N \cdot \phi$

$\theta^{\prime \prime}+\frac{1}{2} f \cdot \theta^{\prime}+D \phi^{\prime \prime}+A^{*} \cdot e^{-\eta}=\frac{1}{2} \xi\left(f^{\prime} \frac{\partial \theta}{\partial \xi}-\theta^{\prime} \frac{\partial f}{\partial \xi}\right)$

$\frac{1}{L e} \phi^{\prime \prime}+\frac{1}{2} f \cdot \phi^{\prime}+S \theta^{\prime \prime}=\frac{1}{2} \xi\left(f^{\prime} \frac{\partial \phi}{\partial \xi}-\phi^{\prime} \frac{\partial f}{\partial \xi}\right)$

The boundary conditions are defined as follows:

$\begin{array}{ll}\eta=0: & f=-\frac{\xi}{2}, \quad \theta=1, \phi=1 \\ \eta \rightarrow \infty: & \theta=0, \quad \phi=0\end{array}$

For the new variables, the Darcian velocities in the $x-$ and $y-$ directions are also respectively obtained by

$\begin{array}{ll}\eta=0: & f=-\frac{\xi}{2}, \\ \eta \rightarrow \infty & \theta, \quad \phi=1 \\ & \theta=0, \quad \phi=0\end{array}$

where primes denote differentiation with respect to $\eta$. $\xi$ defined in Eq. (13.1) is the surface blowing/suction parameter; Eq. (18) can be obtained by integrating Eq. (21) versus $\xi$ once and by setting $\eta=0$ (at the surface, $y=0$, then $\eta=0$ ), and with the help of boundary Eq. (7). On the one hand, for the case of blowing, $V_{w}>0$ and hence $\xi>0$. On the other hand, for the case of suction, $V_{w}<0$ and hence $\xi<0$. Besides, the buoyancy ratio $N$, the Lewis number $L e$, the Dufour parameter $D$ and the Soret parameter $S$ are respectively defined as follows:

$N=\frac{\beta_{C}\left[C_{w}-C_{\infty}\right]}{\beta_{T}\left[T_{w}-T_{\infty}\right]}, \quad L e=\frac{\alpha_{m}}{D_{M}}$

$D=\frac{\bar{D}\left[C_{w}-C_{\infty}\right]}{\alpha_{m}\left[T_{w}-T_{\infty}\right]}, \quad S=\frac{\bar{S}\left[T_{w}-T_{\infty}\right]}{\alpha_{m}\left[C_{w}-C_{\infty}\right]}$

The results for heat and mass transfer rates have practical applications. The heat and mass transfer rates are expressed in terms of the local Nusselt number $N u_{x}$ and the local Sherwood number $S h_{x}$ respectively, which are respectively defined as follows:

$N u_{x}=\frac{h_{x} x}{k}=\frac{q_{w} x}{\left[T_{w}-T_{\infty}\right] \cdot k}=\frac{-\left.\left(\frac{\partial T}{\partial y}\right)\right|_{y=0} x}{\left[T_{w}-T_{\infty}\right]}$

$S h_{x}=\frac{h_{m, x} x}{D_{M}}=\frac{m_{w} x}{\left[C_{w}-C_{\infty}\right] \cdot D_{M}}=\frac{-\left.\left(\frac{\partial C}{\partial y}\right)\right|_{y=0} x}{\left[C_{w}-C_{\infty}\right]}$ 
By applying Eq. (13), the local Nusselt number $N u_{x}$ and the local Sherwood number $S h_{x}$ in terms of $R a_{x}^{1 / 2 n}$ are respectively obtained by

$$
\begin{aligned}
& \frac{N u_{x}}{R a_{x}{ }^{1 / 2 n}}=-\theta^{\prime}(\xi, 0) \\
& \frac{S h_{x}}{R a_{x}{ }^{1 / 2 n}}=-\phi^{\prime}(\xi, 0)
\end{aligned}
$$

It may be noticed that for $\xi=0 \quad$ (without blowing/suction effect), $M=A^{*}=0 \quad$ (without magnetic field and internal heat generation effects) Eqs. (15)-(19) are reduced to those of Tai and Char (2010) where a similar solution was obtained previously.

\section{NUMERICAL METHOD}

Equations (15)-(19) are integrating by combining the implicit finite difference approximation with the modified Keller box method of Cebeci and Bradshaw (1984). First, the partial differential converted into a system of five first-order equations. These first-order equations are then expressed in finite difference forms and solved along with their boundary conditions by applying an iterative scheme. This approach improves the convergence rate and the computation times.
Computations were performed with a personal computer with $\Delta \xi=0.1$ and the first step size $\Delta \eta_{l}=0.01$. The variable grid parameter is chosen 1.01 and the value of $\eta_{\infty}=30$. The iterative procedure is stopped to give the final temperature and concentration distributions when the error in computing the $\theta_{w}^{\prime}$ and $\phi_{w}^{\prime}$ in the next procedure becomes less than $10^{-5}$.

\section{RESULTS AND DISCUSSION}

The accuracy of this method was verified by comparing the results with those of Postelnicu (2004), Hsiao et al. (2014) and Yih and Huang (2015). Table 1 lists the comparison of $-\theta^{\prime}(\xi, 0)$ and $-\phi^{\prime}(\xi, 0)$ for various values of $M, N, D$ and $S$ with $A^{*}=\xi=0, n=L e=1$. Table 2 lists the comparison of $-\theta^{\prime}(\xi, 0)$ and $-\phi^{\prime}(\xi, 0)$ for various values of $A^{*}$ and $n$ with $M=D=S=\xi=0, N=4, L e=10$. All values in Tables 1 to 2 lists the comparisons showed excellent agreement with the numerical data in previous works. Table 3 lists the values of $N u_{x} / R a_{x}^{1 / 2 n}$ and $S h_{x} / R a_{x}^{1 / 2 n}$ for various values of $N, L e$, $D, S, \xi, M, n$ and $A^{*}$.

Table 1. Comparison of $-\theta^{\prime}(\xi, 0)$ and $-\phi^{\prime}(\xi, 0)$ for various values of $M, N, D$ and $S$ with $A^{*}=\xi=0, n=L e=1$.

\begin{tabular}{cccccccccc}
\hline & & & & \multicolumn{3}{c}{$-\theta^{\prime}(\xi, 0)$} & & \multicolumn{2}{c}{$-\phi^{\prime}(\xi, 0)$} \\
\cline { 5 - 9 }$M$ & $N$ & $D$ & $S$ & $\begin{array}{c}\text { Postelnicu } \\
(2004)\end{array}$ & $\begin{array}{c}\text { Hsiao et } \\
\text { al. }(2014)\end{array}$ & $\begin{array}{c}\text { Present } \\
\text { results }\end{array}$ & $\begin{array}{c}\text { Postelnicu } \\
(2004)\end{array}$ & $\begin{array}{c}\text { Hsiao et al. } \\
(2014)\end{array}$ & $\begin{array}{c}\text { Present } \\
\text { results }\end{array}$ \\
\hline 0 & 0.5 & 0.075 & 0.8 & 0.5550 & 0.5550 & 0.5550 & 0.2876 & 0.2876 & 0.2876 \\
1 & 0.5 & 0.075 & 0.8 & 0.3925 & 0.3925 & 0.3925 & 0.2033 & 0.2033 & 0.2034 \\
0 & 1 & 0.03 & 2.0 & 0.7144 & 0.7144 & 0.7144 & -0.1359 & -0.1395 & -0.1395 \\
1 & 1 & 0.03 & 2.0 & 0.5051 & 0.5051 & 0.5051 & -0.0986 & -0.0986 & -0.0986 \\
\hline
\end{tabular}

Table 2. Comparison of $-\theta^{\prime}(\xi, 0)$ and $-\phi^{\prime}(\xi, 0)$ for various values of $A^{*}$ and $n$ with $M=D=S=\xi=0, N=4, L e=10$.

\begin{tabular}{cccccc}
\hline & & \multicolumn{2}{c}{$-\theta^{\prime}(\xi, 0)$} & \multicolumn{2}{c}{$-\phi^{\prime}(\xi, 0)$} \\
\cline { 3 - 6 }$A^{*}$ & $n$ & $\begin{array}{c}\text { Yih and Huang } \\
(2015)\end{array}$ & $\begin{array}{c}\text { Present } \\
\text { results }\end{array}$ & $\begin{array}{c}\text { Yih and Huang } \\
(2015)\end{array}$ & $\begin{array}{c}\text { Present } \\
\text { results }\end{array}$ \\
\hline \multirow{2}{*}{0} & 0.5 & 1.0105 & 1.0104 & 6.3671 & 6.3671 \\
& 1.0 & 0.6811 & 0.6810 & 3.2892 & 3.2892 \\
& 2.0 & 0.6030 & 0.6029 & 2.4022 & 2.4022 \\
\hline \multirow{2}{*}{1} & 0.5 & 0.2404 & 0.2402 & 6.4412 & 6.4412 \\
& 1.0 & -0.0191 & -0.0191 & 3.3311 & 3.3311 \\
& 2.0 & -0.0837 & -0.0837 & 2.4247 & 2.4247 \\
\hline
\end{tabular}

Table 3. Values of $N u_{x} / R a_{x}^{1 / 2 n}$ and $S h_{x} / R a_{x}^{1 / 2 n}$ for various values of $N, L e, D, S, \xi, M, n$ and $A^{*}$.

\begin{tabular}{cccccccccc}
\hline$N$ & $L e$ & $D$ & $S$ & $\xi$ & $M$ & $n$ & $A^{*}$ & $N u_{x} / R a_{x}^{1 / 2}$ & $S h_{x} / R a_{x}^{1 / 2}$ \\
\hline 1 & 1 & 0 & 0 & 0 & 0 & 1 & 0 & 0.6275 & 0.6275 \\
1 & 1 & 0.1 & 0.1 & 0 & 0 & 1 & 0 & 0.5983 & 0.5983 \\
0 & 1 & 0.1 & 0.1 & 0 & 0 & 1 & 0 & 0.4230 & 0.4230 \\
1 & 2 & 0.1 & 0.1 & 0 & 0 & 1 & 0 & 0.5435 & 0.8849 \\
1 & 1 & 0.2 & 0.1 & 0 & 0 & 1 & 0 & 0.5638 & 0.6053 \\
1 & 1 & 0.1 & 0.2 & 0 & 0 & 1 & 0 & 0.6053 & 0.5638 \\
1 & 1 & 0.1 & 0.1 & 1 & 0 & 1 & 0 & 0.4197 & 0.4197 \\
1 & 1 & 0.1 & 0.1 & -1 & 0 & 1 & 0 & 0.8284 & 0.8284 \\
1 & 1 & 0.1 & 0.1 & 0 & 1 & 1 & 0 & 0.4230 & 0.4230 \\
1 & 1 & 0.1 & 0.1 & 0 & 0 & 0.5 & 0 & 0.7179 & 0.7179 \\
1 & 1 & 0.1 & 0.1 & 1 & 0 & 1.5 & 0 & 0.5710 & 0.5710 \\
1 & 1 & 0.1 & 0.1 & 0 & 0 & 1 & 1 & -0.0725 & 0.7022 \\
\hline
\end{tabular}


The numerical results are presented for the buoyancy ratio $N=5$, the Lewis number $L e=2$, the Dufour parameter $D=0.1$, the Soret parameter $S=0.2$, the power-law index of the non-Newtonian fluid $n$ ranging from 0.5 to 3.0 , the blowing/suction parameter $\xi$ ranging from -1 to 1, the magnetic field parameter $M$ ranging from 0 to 2 and the internal heat generation $A^{*}$ ranging from 0 to 1 . (a)

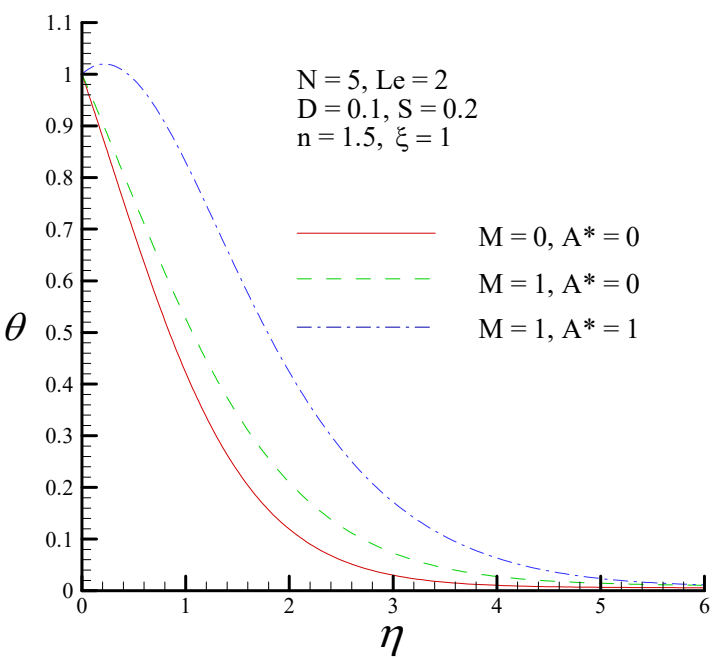

(b)

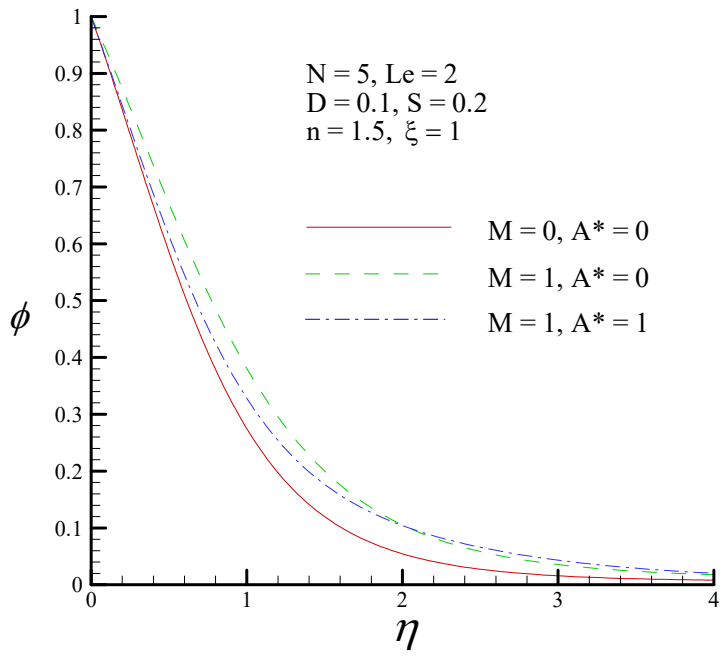

Fig. 2 (a) The dimensionless temperature profile and (b) the dimensionless concentration profile for two values of $M$ and $A^{*}$.

Table 4. The values of $N u_{x} / R a_{x}^{1 / 2 n}$ and $S h_{x} / R a_{x}^{1 / 2 n}$ for various values of $M$ and $A^{*}$ with $N=5, L e=2, D=0.1, S=0.2, n=1.5, \xi=1$.

\begin{tabular}{ccccccc}
\hline & \multicolumn{3}{c}{$N u_{x} / R a_{x}^{1 / 2 n}$} & \multicolumn{3}{c}{$S h_{x} / R a_{x}^{1 / 2 n}$} \\
\cline { 2 - 7 } & $A^{*}=0.0$ & $A^{*}=0.5$ & $A^{*}=1.0$ & $A^{*}=0.0$ & $A^{*}=0.5$ & $A^{*}=1.0$ \\
\hline 0.0 & 0.5894 & 0.2794 & -0.0268 & 0.7686 & 0.8489 & 0.9282 \\
0.5 & 0.5161 & 0.1992 & -0.1139 & 0.6694 & 0.7511 & 0.8321 \\
1.0 & 0.4582 & 0.1363 & -0.1820 & 0.5897 & 0.6725 & 0.7545 \\
1.5 & 0.4116 & 0.0860 & -0.2363 & 0.5250 & 0.6085 & 0.6913 \\
2.0 & 0.3734 & 0.0449 & -0.2806 & 0.4710 & 0.5557 & 0.6390 \\
\hline
\end{tabular}

Figure 2 illustrates the two values of the magnetic field parameter $M \quad(M=0,1)$ and the internal heat generation coefficient $A^{*} \quad\left(A^{*}=\right.$ $0,1)$ on the dimensionless temperature profile and concentration profile with $N=5, L e=2, D=0.1, S=0.2, n=1.5, \quad \xi=1$, respectively. These two figures show that the dimensionless temperature and concentration profiles decrease monotonically from the surface of the vertical flat plate to the ambient except the case of $M=1, A^{*}=1$. Increasing the magnetic field parameter or the internal heat generation coefficient worsen the dimensionless wall temperature gradient, as depicted in Fig. 2(a). The dimensionless wall concentration gradient enhances with a decrease in the magnetic field parameter or the internal heat generation coefficient, as illustrated in Fig. 2(b). Besides, increasing internal heat generation coefficient $A^{*}$ from 0 to 1 causes the dimensionless wall temperature gradient $-\theta^{\prime}(\xi, 0)$ to become negative value. This phenomenon is due to overshoot in the dimensionless temperature profile for $M=1, A^{*}=1$ where heat transfer is from the porous medium to the vertical flat plate.

Table 4 lists the values of local Nusselt number $N u_{x} / R a_{x}^{1 / 2 n}$ and the local Sherwood number $S h_{x} / R a_{x}^{1 / 2 n}$ for various values of $M$ and
$A^{*}$ with $N=5, L e=2, D=0.1, S=0.2, n=1.5, \xi=1$. First, for fixed $M$, as the internal heat generation coefficient $A^{*}$ is enhanced, the local Nusselt number decreases but the local Sherwood number increases. This is because increasing the internal heat generation coefficient $A^{*}$ reduces the dimensionless surface temperature gradient $-\theta^{\prime}(\xi, 0)$ and increases the dimensionless surface concentration gradient $-\phi^{\prime}(\xi, 0)$, as displayed in Fig. 2. Second, for fixed $A^{*}$, the local Nusselt number and the local Sherwood number tends to decrease as the magnetic field parameter $M$ is increased. Increasing the magnetic field parameter increases the thermal boundary layer thickness and the concentration boundary layer thickness, as revealed in Fig. 2. The thicker the thermal concentration boundary layer thickness, the smaller the local Nusselt (Sherwood) number.

To determine the effects of step size $\Delta \xi$ (the first step size $\left.\Delta \eta_{1}\right)$, the numerical code for the model was run with three different step sizes as $\Delta \xi=0.2,0.1$ and $0.05\left(\Delta \eta_{1}=0.05,0.01\right.$ and 0.005$)$. The grid independency is done as shown in Fig. 3, for the case of $N=5$, $L e=2, D=0.1, S=0.2, n=1.5, \xi=1, M=1, A^{*}=0.0$. 
(a)

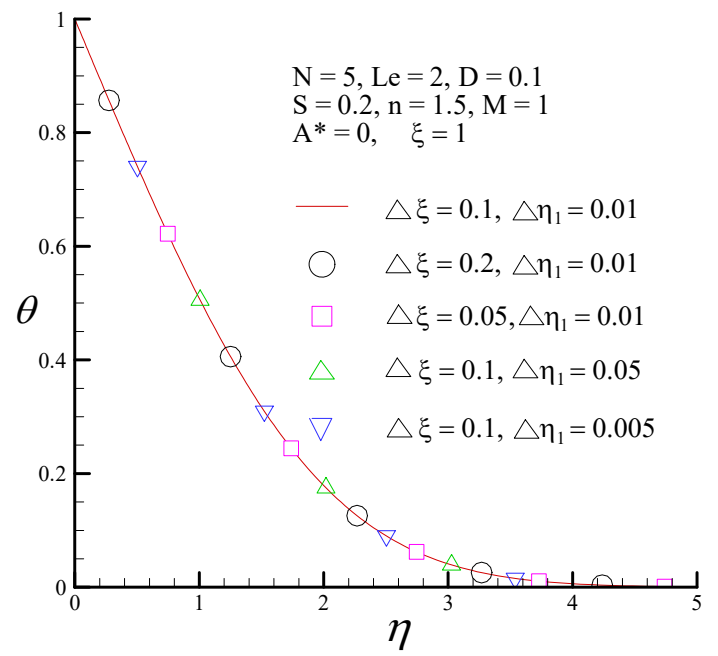

(b)

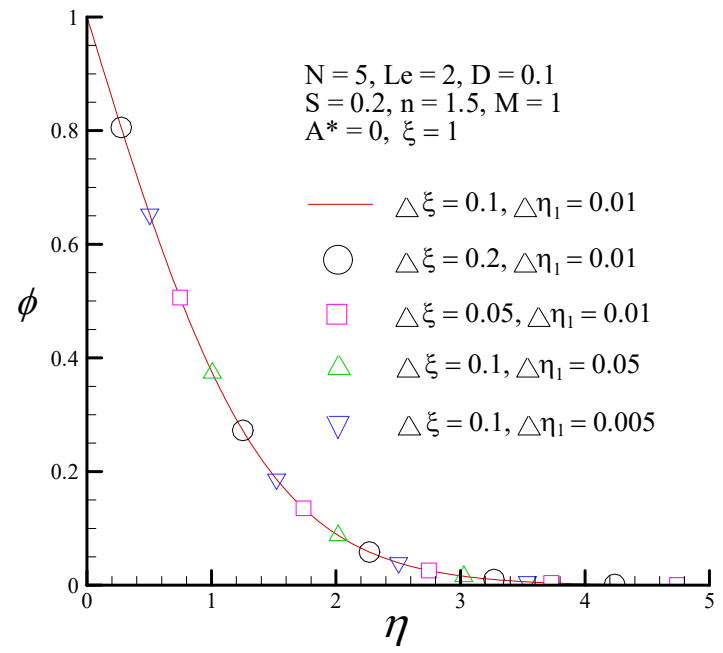

Fig. 3 (a) The dimensionless temperature profile and (b) the dimensionless concentration profile for the case of $N=5, L e=2$, $D=0.1, S=0.2, n=1.5, \xi=1, M=1, A^{*}=0.0$.

$(a)$

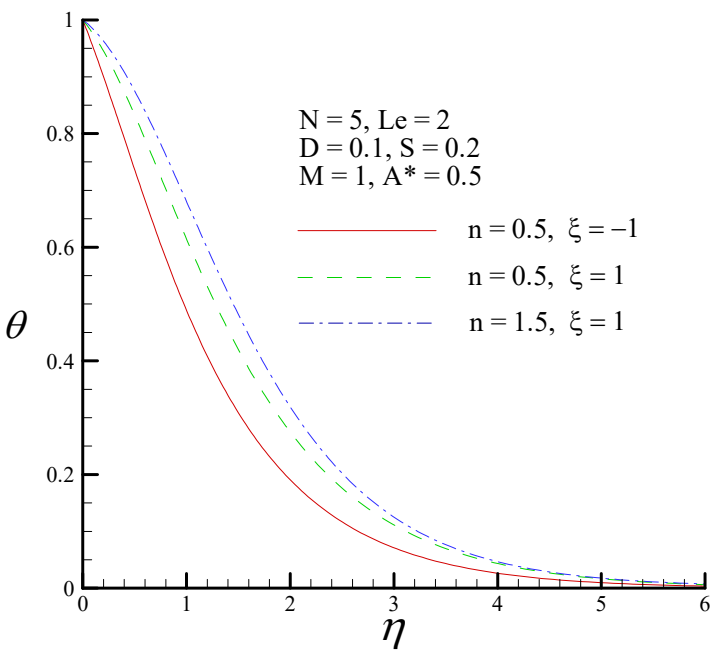

(b)

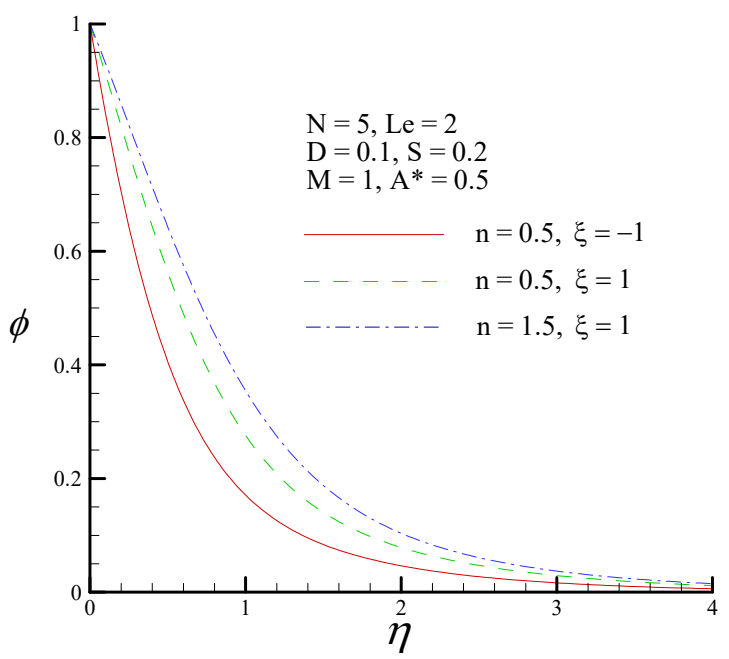

Fig. 4 (a) The dimensionless temperature profile and (b) the dimensionless concentration profile for two values of $n$ and $\xi$.

Table 5. The values of $N u_{x} / R a_{x}^{1 / 2 n}$ and $S h_{x} / R a_{x}^{1 / 2 n}$ for various values of $n$ and $\xi$ with $N=5, L e=2$, $D=0.1, S=0.2, M=1, A^{*}=0.5$.

\begin{tabular}{ccccccc}
\hline \multirow{3}{*}{$n$} & \multicolumn{3}{c}{$N u_{x} / R a_{x}^{1 / 2 n}$} & \multicolumn{3}{c}{$S h_{x} / R a_{x}^{1 / 2 n}$} \\
\cline { 2 - 7 } & $\xi=-1$ & $\xi=0$ & $\xi=1$ & $\xi=-1$ & $\xi=0$ & $\xi=1$ \\
\hline 0.5 & 0.4616 & 0.3259 & 0.2224 & 1.6720 & 1.2173 & 0.8613 \\
0.8 & 0.4371 & 0.2974 & 0.1919 & 1.6118 & 1.1488 & 0.7914 \\
1.0 & 0.4222 & 0.2800 & 0.1730 & 1.5774 & 1.1088 & 0.7502 \\
1.5 & 0.3945 & 0.2465 & 0.1363 & 1.5144 & 1.0339 & 0.6725 \\
2.0 & 0.3773 & 0.2248 & 0.1120 & 1.4756 & 0.9864 & 0.6228 \\
2.5 & 0.3662 & 0.2103 & 0.0956 & 1.4506 & 0.9552 & 0.5902 \\
3.0 & 0.3588 & 0.2002 & 0.0839 & 1.4335 & 0.9335 & 0.5674 \\
\hline
\end{tabular}

Figure 4 presents the two values of the non-Newtonian fluid power-law index $n \quad(n=0.5,1.5)$ and the blowing/suction parameter $\xi(\xi=-1,1)$ on the dimensionless temperature and concentration profiles with $N=5, L e=2, D=0.1, \quad S=0.2, \quad M=1, A^{*}=0.5$, respectively. It is found that the dimensionless temperature profiles $\theta$ and the dimensionless concentration profiles $\phi$ increase with increasing the non-Newtonian fluid power-law index $n$, thus increasing the thermal and concentration boundary layer thicknesses. This is because increasing the non-Newtonian fluid power-law index $n$ tends to decrease the buoyancy force and slow the flow velocity. Therefore, pseudoplastic fluids $(n=0.5)$ are superior to the dilatant fluids ( $n=1.5$ ) from the viewpoint of the heat and mass transfer rates by free convection from a vertical plate embedded in a porous medium 
saturated with non-Newtonian power-law fluids.

Table 5 lists the values of local Nusselt number $N u_{x} / R a_{x}^{1 / 2 n}$ and the local Sherwood number $S h_{x} / R a_{x}^{1 / 2 n}$ for various values of $n$ and $\xi$ with $N=5, L e=2, D=0.1, S=0.2, M=1, A^{*}=0.5$. It is found that for the fixed $n$, enhancing the blowing/suction parameter $\xi$ has the tendency to reduces both the local Nusselt number and the local Sherwood number. This is because for the case of blowing decreases both the dimensionless surface temperature and concentration gradients, as shown in Fig. 3. However, this trend reversed for suction of fluid.

\section{Conclusions}

A two-dimensional laminar boundary layer analysis is presented to study the influence of uniform blowing/suction and MHD on the free convection of non-Newtonian fluids over a vertical plate in porous media with Soret/Dufour effect. After the coordinate transformation, the transformed governing equations are solved by Keller box method (KBM). Comparisons with previously published works show excellent agreement. Numerical solutions are obtained for different values of the blowing/suction parameter $\xi$, the magnetic field parameter $M$, the power-law index of the non-Newtonian fluid $n$ and the internal heat generation coefficient $A^{*}$. The calculations show that, when the magnetic field parameter $M$ and the non-Newtonian fluid $n$ increase, both the local Nusselt number and the local Sherwood number decrease. Otherwise, increasing the internal heat generation coefficient $A^{*}$ reduces the local Nusselt number and increases the local Sherwood number. In the case of suction, both the local Nusselt number and the local Sherwood number increase. Blowing has the opposite effect.

\section{NOMENCLATURE}

$\begin{array}{ll}A^{*} & \text { internal heat generation coefficient } \\ B_{o} & \text { externally imposed magnetic field in the y-direction } \\ C & \text { concentration } \\ C_{P} & \text { specific heat at constant pressure } \\ D & \text { Dufour parameter } \\ d & \text { particle diameter } \\ \bar{D} & \text { Dufour coefficient } \\ D_{M} & \text { mass diffusivity } \\ f & \text { dimensionless stream function } \\ g & \text { gravitational acceleration } \\ h_{x} & \text { local convective heat transfer coefficient } \\ h_{m, x} & \text { local convective mass transfer coefficient } \\ K & \text { permeability of the porous medium } \\ k & \text { equivalent thermal conductivity } \\ L e & \text { Lewis number } \\ M & \text { magnetic field parameter } \\ N & \text { buoyancy ratio } \\ N u_{x} & \text { local Nusselt number } \\ m_{w} & \text { local mass flux } \\ n & \text { power-law index of the non-Newtonian fluid } \\ q_{w} & \text { local heat flux } \\ q^{\prime \prime \prime} & \text { internal heat generation rate per unit volume } \\ R a_{x} & \text { modified local Rayleigh number } \\ S & \text { Soret parameter } \\ \bar{S} & \text { local Sherwood number } \\ S h_{x} & \end{array}$

$\begin{array}{ll}T & \text { temperature } \\ u & \text { Darcy velocity component in the } x \text {-direction } \\ v & \text { Darcy velocity component in the } y \text {-direction } \\ V_{w} & \text { uniform transpiration velocity } \\ x & \text { streamwise coordinate } \\ y & \text { transverse coordinate } \\ \text { Greek symbols } & \\ \alpha_{m} & \text { equivalent thermal diffusivity } \\ \beta_{C} & \text { coefficient of concentration expansion } \\ \beta_{T} & \text { coefficient of thermal expansion } \\ \varepsilon & \text { porosity } \\ \eta & \text { pseudo-similarity variable } \\ \theta & \text { dimensionless temperature } \\ \mu & \text { absolute viscosity of fluid } \\ \rho & \text { density of fluid } \\ \sigma & \text { electric conductivity of the fluid } \\ \phi & \text { dimensionless concentration } \\ \psi & \text { stream function } \\ \text { Subscripts } & \\ w & \text { condition at the wall } \\ \infty & \text { condition at infinity }\end{array}$

\section{References}

Nield, D.A., and Bejan, A., 2006, Convection in Porous Media, Springer-Verlag, New York.

Shenoy, A.V., 1994, "Non-Newtonian Fluid Heat Transfer in Porous Media," Advances in Heat Transfer, 24, 102-184. https://doi.org/10.1016/S0065-2717(08)70233-8

Chen, H.T., and Chen, C.K., 1988, "Free Convection of NonNewtonian Fluids along a Vertical Plate Embedded in a Porous Medium," ASME Journal of Heat Transfer, 110(1), 257-260.

https://doi.org/10.1115/1.3250462

Yang, Y.T., and Wang, S.J., 1996, "Free Convection Heat Transfer of Non-Newtonian Fluids over Axisymmetric and Two-Dimensional Bodies of Arbitrary Shape Embedded in a Fluid-Saturated Porous Medium," International Journal of Heat and Mass Transfer, 39(1), 203-210. https://doi.org/10.1016/S0017-9310(96)85016-2

Gorla, R.S.R., and Kumari, M., 1998 "Nonsimilar Solutions for Free Convection in Non-Newtonian Fluids along a Vertical Plate in a Porous Medium," Transport in Porous Media, 33(3), 295-307.

https://doi.org/10.1023/A:1006566004291

Rastogi, S.K., and Poulikakos, D., 1995, "Double-Diffusion from a Vertical Surface in a Porous Region Saturated with a Non-Newtonian Fluid," International Journal of Heat and Mass Transfer, 38(5), 935-946. https://doi.org/10.1016/0017-9310(94)00198-5

Jumah, R.Y., and Mujumdar, A.S., 2000, "Free Convection Heat and Mass Transfer from a Vertical Plate with Variable Wall Temperature and Concentration to Power Law Fluids with Yield Stress in a Porous Medium," Chemical Engineering Communications, 185(1), 165-182. https://doi.org/10.1016/S0735-1933(00)00131-7

Cheng, C.Y., 2006, "Natural Convection Heat and Mass Transfer of Non-Newtonian Power Law Fluids with Yield Stress in Porous Media from a Vertical Plate with Variable Wall Heat and Mass Fluxes," International Communications in Heat and Mass Transfer, 33(9), 1156- 
1164.

https://doi.org/10.1016/j.icheatmasstransfer.2006.06.006

Tai, B.C., and Char, M.I., 2010, "Soret and Dufour Effects on Free Convection Flow of Non-Newtonian Fluids along a Vertical Plate Embedded in a Porous Medium with Thermal Radiation," International Communications in Heat and Mass Transfer, 37(5), 480-483.

https://doi.org/10.1016/j.icheatmasstransfer.2009.12.017

Yih, K.A., and Huang, C.J., 2015, "Effect of Internal Heat Generation on Free Convection Flow of Non-Newtonian Fluids over a Vertical Truncated Cone in Porous Media: VWT/VWC," Journal of Air Force Institute Technology, 14, 1-18.

https://doi.org/10.6125/15-0316-844

Postelnicu, A., 2004, "Influence of a Magnetic field on Heat and Mass Transfer by Natural Convection from Vertical Surfaces in Porous media Considering Soret and Dufour Effects," International Journal of Heat and Mass Transfer, 47(6-7), 1467-1472.

https://doi.org/10.1016/j.ijheatmasstransfer.2003.09.017

Partha, M.K., Murthy, P.V.S.N., and Raja Sekhar, G.P., 2006, "Soret and Dufour Effects in a Non-Darcy Porous Medium," ASME Journal of Heat Transfer, 128(6), 605-610.

https://doi.org/10.1115/1.2188512

Cheng, C.Y., 2007, "Double Diffusion from a Vertical Wavy Surface in a Porous Medium Saturated with a Non-Newtonian Fluid," International Communications in Heat and Mass Transfer, 34(3), 285294.

https://doi.org/10.1016/j.icheatmasstransfer.2006.09.006

Hsiao, C.Y., Chang, W.J., Char, M.I., and Tai, B.C., 2014, "Influence of Thermophoretic Particle Deposition on MHD Free Convection Flow of Non-Newtonian Fluids from a Vertical Plate Embedded in Porous Media Considering Soret and Dufour Effects," Applied Mathematics and Computation, 244, 390-397.

https://doi.org/10.1016/j.amc.2014.07.007

Crepeau, J.C., and Clarksean, R., 1997, "Similarity Solutions of Natural Convection with Internal Heat Generation," ASME Journal of Heat Transfer, 119(1), 183-185.

https://doi.org/10.1115/1.2824086

Postelnicu, A., and Pop, I., 1999, "Similarity Solutions of Free Convection Boundary Layers over Vertical and Horizontal Surfaces in Porous Media with Internal Heat Generation," International Communications in Heat and Mass Transfer, 26(8), 1183-1191. https://doi.org/10.1016/S0735-1933(99)00108-6

Grosan, T., and Pop, I., 2001, "Free Convection over Vertical Flat Plate with a Variable Wall Temperature and Internal Heat Generation in a Porous Medium Saturated with a Non-Newtonian Fluid," Technische Mechanik, 21, 313-318.

Grosan, T., Revnic, C., Pop, I., and Ingham, D.B., 2009, "Magnetic Field and Internal Heat Generation Effects on the Free Convection in a Rectangular Cavity Filled with a Porous Medium," International Journal of Heat and Mass Transfer, 52(5-6), 1525-1533.

https://doi.org/10.1016/j.ijheatmasstransfer.2008.08.011

Minkowycz, W.J., and Cheng, P., 1982, "Local Non-Similar Solutions for Free Convective Flow with Uniform Lateral Mass Flux in Porous Medium," Letters in Heat and Mass Transfer, 9(3), 159-168.

https://doi.org/10.1016/0094-4548(82)90054-6

Yih, K.A., 1997, “The Effect of Uniform Lateral Mass Flux on Free
Convection about a Vertical Cone Embedded in a Saturated Porous Medium," International Communications in Heat and Mass Transfer, 24(8), 1195-1205. https://doi.org/10.1016/S0735-1933(97)00114-0

Yih, K.A., 1998, "Uniform Lateral Mass Flux Effect on Natural Convection of Non-Newtonian Fluids over a Cone in Porous Media," International Communications in Heat and Mass Transfer, 25(7), 959968.

https://doi.org/10.1016/S0735-1933(98)00087-6

Chamkha, A.J., and Ben-Nakhi, A., 2008, "MHD Mixed ConvectionRadiation Interaction along a Permeable Surface Immersed in a Porous Medium in the Presence of Soret and Dufour's Effects," Heat and Mass Transfer, 44, 846-856.

https://doi.org/10.1007/s00231-007-0296-x

Kumari, M., and Nath, G., 2009, "Natural Convection from a Vertical Cone in a Porous Medium due to the Combined Effects of Heat and Mass Diffusion with Non-Uniform Wall Temperature/Concentration or Heat/Mass Flux and Suction/Injection," International Journal of Heat and Mass Transfer, 52(13-14), 3064-3069.

https://doi.org/10.1016/j.ijheatmasstransfer.2008.10.037

Rashad, A.M., EL-Hakiem, M.A., and Abdou, M.M.M., 2011, "Natural Convection Boundary Layer of a Non-Newtonian Fluid about a Permeable Vertical Cone Embedded in a Porous Medium Saturated with a Nanofluid," Computers and Mathematics with Applications, 62(8), 3140-3151.

https://doi.org/10.1016/i.camwa.2011.08.027

Huang, C.J., 2017, "Influence of Uniform Blowing/Suction on the Free Convection of Non-Newtonian Fluids over a Vertical Cone in Porous Media with Thermal Radiation and Soret/Dufour Effects: Uniform Wall Temperature/Uniform Wall Concentration," ASME Journal of Heat Transfer, 139(3), 032501. https://doi.org/10.1115/1.4035041

Huang, C.J., 2018, "Effects of Internal Heat Generation and Soret/Dufour on Natural Convection of Non-Newtonian Fluids over a Vertical Permeable Cone in a Porous Medium," Journal of King Saud University-Science, 30(1), 106-111. https://doi.org/10.1016/j.jksus.2016.09.009

Christopher, R.V., and Middleman, S., 1965, "Power-Law Flow through a Packed Tube," Industry and Engineering Chemistry Fundamentals, 4(4), 424-426. https://doi.org/10.1021/i160016a011

Dharmadhirkari, R.V., and Kale, D.D., 1985, "Flow of Non-Newtonian Fluids through Porous Media," Chemical Engineering Science, 40(3), 527-529.

https://doi.org/10.2118/7150-PA

Cebeci, T., and Bradshaw, P., 1984, Physical and Computational Aspects of Convective Heat Transfer, Springer-Verlag, New York.

Hooper, W.B., Chen, T.S., and Armaly, B.F., 1994, "Mixed Convection from a Vertical Plate in Porous Media with Surface Injection or Suction," Numerical Heat Transfer, Part A: Applications, 25(3), 317329.

https://doi.org/10.1080/10407789408955951

Hong, J.T., Yamada, Y., and Tien, C.L., 1987, "Effects of Non-Darcian and Non-Uniform Porosity on Vertical Plate-Natural Convection in Porous Media," ASME Journal of Heat Transfer, 109(2), 356-362. https://doi.org/10.1115/1.3248088 